\title{
Microbial diversity in the hot spring Faust Lake, Kunashir Island
}

\author{
A. Rozanov ${ }^{1 *}$, A. Korzhuk ${ }^{1,2}$, S. Peltek ${ }^{1}$ \\ ${ }^{1}$ Institute of Cytology and Genetics SB RAS, Novosibirsk, Russia \\ ${ }^{2}$ Novosibirsk State University, Novosibirsk, Russia \\ *e-mail: sibiryak.n@gmail.com
}

Key words: metagenomic, hot spring, microbial communities, genome

Motivation and Aim: Metagenomics is the study of genetic material recovered directly from environmental samples. This approach makes it possible to obtain information about uncultivated microorganisms. Microbial communities of extreme ecosystems have an applied and fundamental interest. Applied interest is mainly focused on searching for new protein sequences. From the fundamental point of view communities of extreme ecosystems are interesting as simpler models, in comparison with communities of usual ecosystems. But the greatest interest now is caused by the fact that communities of extreme ecosystems are living chronicles that contain information about microbial communities of long-past geological epochs.

Kunashir is one of the southern islands of the Kurile-Kamchatka volcanic belt located in Russia. The large amount of organic remains from the environment that fall into water sources are favorable for the microflora grows. Its makes hot springs on the island were interestin for microbiologist. Due to the closeness of the territory, the geothermal springs of the southern Kuril Islands, were not studied by microbiology methods and with the metagenomic approaches.

Methods and Algorithms: In the summer of 2017, researchers from the Laboratory of Molecular Biotechnologies of the IC\&G SB RAS organized the expedition to collect material for metagenomic research. To date, we have performed a metagenomic sequencing of microbial communities from the bottom sediments of Faust Lake. Libraries for sequencing were prepared by Ph.D. Vasilyev G.V. in the Center for Genomic Research of the IC\&G SB RAS. Sequencing was carried out by Genetics and Reproductive Medicine Center "Genetico" (Moscow) on the Illumina NovaSeq 6000.

Results: The data allows extracting most of the genomic sequences for prokaryotes with abundance greater than $0.1 \%$ of the total number of microorganisms. During the treatment, we obtained data about a fairly complete sequence of new Cyanidioschyzon species. It is unicellular haploid red alga adapted to high sulfur acidic hot spring environments. We have data about genomes of new bacterial species with different complitnes. Previously, similar work in Russia was not performed.

Conclusion: The results obtained in the work will give a new material for phylogeography of microorganisms and also will allow obtaining data on the genomes of previously unknown microorganisms.

Acknowledgements: Supported by the fundamental research program of the SB RAS II.1 No. 0324-2018-0039. 